\title{
The Effect of Forage-Based Meat Goat Production Systems on Live Performance, Carcass Traits and Fatty Acid Composition of Kiko Crossbred Goats
}

\author{
Min BR* , Solaiman S, Gurung N and McElhenney W \\ Department of Agricultural and Environmental Sciences, Tuskegee University, \\ Tuskegee, AL, USA
}

*Corresponding author: Min BR, Department of Agricultural and Environmental Sciences, Tuskegee University, Tuskegee, AL, USA, Tel: +1 334524 7670; E-mail: minb@mytu.tuskegee.edu

Rec date: Nov 02, 2015, Acc date: Dec 14, 2015, Pub date: Dec 18, 2015

Copyright: (C) 2015 Min BR, et al. This is an open-access article distributed under the terms of the Creative Commons Attribution License, which permits unrestricted use, distribution, and reproduction in any medium, provided the original author and source are credited.

\section{Abstract}

It is hypothesize that combining legume forages with grasses will increase plant protein output as well as animal performance. Two years of winter grazing experiments were conducted at the Caprine Research and Education Unit at George Washington Carver Agricultural Experiment Station, Tuskegee University to develop a profitable and sustainable forage-based meat goats production system. Forty-eight Kikocrossbred (Capra hircus; 19.6 \pm 1.5 ) meat goats were randomly allocated to 6 groups as following: (1) annual ryegrass/wheat (RW; Lolium multiflorum/Triticum aestivum, respectively) + Berseem clover (BC; Trifolium alexandrinum), (2) RW + Austrian pea (AP; Pisum sativum), (3) RW + Hairy vetch (HV; Vicia villosa) + AP, (4) RW + HV + AP + BC, (5) RW + HV, and (6) annual RW as a control with 2 replicates per treatment during 2 years. An average multi-culture forage dry matter (DM; $\mathrm{kg} / \mathrm{DM} / \mathrm{ha}$ ) production during 2 years tended to be $(p=0.07)$ greater for $R W+B C$ than other forage combinations. Biomass production in February and April were higher $(\mathrm{P}<0.01)$ for $\mathrm{RW}+\mathrm{BC}$ than $\mathrm{RW}+\mathrm{HV}$ and RW forage combinations, but there was no forage treatment $x$ year interactions. There were no differences in initial body weight (BW), final BW and average daily gain (ADG) among treatments, with no treatment $x$ year interactions for animal BW changes and ADG. However, hot carcass weight, cold carcass weight, and dressing percentage were greater $(P<0.05)$ for $\mathrm{RW}+\mathrm{BC}$ and $\mathrm{RW}+\mathrm{HV}+\mathrm{AP}$ than for $\mathrm{RW}$ grasses. Sirloin weight was greater $(P<0.04)$ for $R W+B C$ than for RW pasture, while leg weight was greater $(P<0.03)$ for $\mathrm{RW}+\mathrm{HV}+\mathrm{AP}$ than for RW control. Goats grazed on grasses-based diet (RW), had higher average saturated fatty acids (SFA; $P<0.01$ ) and polyunsaturated fatty acids (PUFA; $P<0.05$ ) in intramuscular fat content, but both were lower in mescentric kidney fat (MKF) and subcutaneous fat $(P<0.05-0.01)$ compared to legume forage-based diets. Goats grazed on grass-based diets, had higher omega- 3 and -6 fatty acids in intramuscular fat content $(P<0.05-0.08)$, but both were lower in subcutaneous fat $(P<0.05-0.01)$ compared to legume forage-based diets. This is probably due to higher nutritive values of forage diets throughout the winter grazing period. The results produced indicated a greater increase in carcass yield with more desirable fatty acids compositions in intramuscular fat content in meat goats from grass-legume multiple combinations compared to mono-culture forage.

Keywords Meat goats, Performance, Carcass, Winter forages, Fatty acids, legumes

\section{Introduction}

Forage-based meat goat production system is feasible in Alabama and Southern USA through utilization of cool season and warm season grasses and legumes. This can be achieved by allowing for as much grazing as possible throughout the year. Producers should select a combination of forages with different growth cycles that will best support the objectives of their operation. However, forages differ in their seasonal ability to produce biomass production. Considerable research has been conducted over the years on winter [1-3] and summer [4] forages grazing systems for beef cattle production. However, optimum forage-based grazing system for winter grazing with multi-combinations of grass and legume forage for meat goat production and their carcass evaluation are not well established. 
The effects of various forages feeding on beef composition have been studied for decades. Formerly, the interest was focused mainly on feeding efficiency, gross composition and sensory attributes of beef. Extensive research data show that beef from animals finished on green forages and silages (except for maize silage) has a lower content of intramuscular fat, but a higher proportion of $n-3$ polyunsaturated fatty acids (PUFAs), ratio $n-6: n-3$ PUFAs, vitamin $E$ and $\beta$-carotene, than meat from animals fed on grain-based diets [5]. The role of meat as a source of dietary insufficient n-3 PUFAs has been reviewed by [6]. Besides fatty acid composition of intramuscular fat, the type of forage fed affects the level of some nutritionally desirable beef constituents (e.g. PUFA) [4]. Moreover, the forage species affecting beef fatty acids compositions have been of concern [7]. At present, no information, with meat goats, on the efficacy of using grass/ legume-based finishing systems to increase carcass weight, while maintaining desirable carcass quality and composition, has been published. Therefore, the objective of this study was to evaluate how grasses and grass with legumes forage combinations grazed during the winter months alter live animal performance, animal health, carcass quality, and fatty acid composition in finishing meat goats.

\section{Materials and Methods}

\section{Experimental design}

Two years grazing experiments were conducted during winter month to test forage biomass production, animal performance, carcass yield, and carcass quality analyses. Forty-

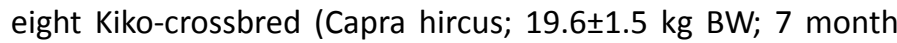
old) meat goats were randomly allocated to 6 groups as following: (1) annual ryegrass/wheat (RW;) + Berseem clover (BC;), (2) RW + Austrian pea (AP;Pisum sativum), (3) RW +
Hairy vetch (HV; ) + AP, (4) RW + HV + AP + BC, (5) RW + HV, and (6) annual RW as a control with 2 replicates per treatment during 2 years. The goats were divided into the groups after there were weaned. A grazing study investigated effects of different grass and legume forages combinations on forage biomass production, animal performance, carcass characteristics, and carcass fatty acid profiles in pastured Kikocrossbred meat goats. Research was conducted on twelve, 0.5ac paddocks at Tuskegee University, Tuskegee, AL.

\section{Pasture and animals}

For this project, six different forage combinations (grasses and legumes) were planted during 2 years in October of 2011 and 2012 for winter grazing. Twelve established paddocks, 0.5 acre each was planted, where two paddocks represented each grass/legume combination. Forage samples were collected monthly starting in February 20 through May 13 of 2011 and 2012 (83 days) to determine forage biomass production with animals grazing ( $n=4 /$ paddock). Herbage mass and forage allowance were measured at $14-$ and $35-d$ intervals. Forage allowance was estimated by hand-clipping winter forages standing crop from five, $1 \mathrm{~m} 2$ quadrats/paddock to ground level.

The experimental paddocks were cultivated and fertilized with $56 \mathrm{~kg}$ of N/ha and $17.8 \mathrm{~kg}$ of S/ha on February 2011 and 2012. On October 2011 and 2012, paddocks were cultivated and sown to multi-forage species (Table 1). Animal BW, forage and fecal samples were taken at monthly intervals. Goats were dosed orally with $0.5 \mathrm{mg} / \mathrm{kg}$ of BW of with Cydectin (Moxidectin; Gene Field Road St. Joseph, Missouri) before experiment commenced. All goats grazed continuously throughout the whole experimental period. Soil samples were taken before and after experiment commenced.

Table 1: Forage biomass production (kg DM/ha) in multi-forage system grazing in meat goats.

\begin{tabular}{|c|c|c|c|c|c|c|c|c|c|c|}
\hline \multirow[t]{2}{*}{ Item } & \multirow[t]{2}{*}{ Year } & \multicolumn{7}{|c|}{ Treatments (Tret) } & \multirow{2}{*}{$\begin{array}{c}\text { p- } \\
\text { value }\end{array}$} & \multirow{2}{*}{$\begin{array}{l}\text { Tret } x \text { year } \\
\text { interaction }\end{array}$} \\
\hline & & A & B & C & D & $E$ & $\mathbf{F}$ & SEM & & \\
\hline \multirow[t]{3}{*}{ February } & 2012 & $841.2 a$ & $635.0 \mathrm{~b}$ & $613.8 \mathrm{~b}$ & $602.5 b$ & $493.8 b$ & $476.3 \mathrm{c}$ & 137.43 & & \\
\hline & 2013 & $1802.0 \mathrm{a}$ & 1330.1 & 225.0 & 1197.5 & $987.5 b$ & $952.5 b$ & 44.84 & & \\
\hline & Mean & $1321.6 a$ & 982.5 & 919.4 & 900.0 & $740.6 \mathrm{~b}$ & $14.4 \mathrm{~b}$ & 97.18 & 0.01 & 0.57 \\
\hline \multirow[t]{3}{*}{ March } & 2012 & 1535.0 & 782.5 & 1842.5 & 875.0 & 1365.0 & 1482.5 & 309.07 & & \\
\hline & 2013 & 3155.5 & 3551.8 & 3510.0 & 3417.5 & 2730.0 & 2965.0 & 319.92 & & \\
\hline & Mean & 2345.2 & 2667.1 & 2676.2 & 2646.3 & 2047.5 & 2223.7 & 218.55 & 0.26 & 0.98 \\
\hline \multirow[t]{3}{*}{ April } & 2012 & $3125.0 a$ & 2630.0 & 2380.0 & 2617.5 & $1952.5 b$ & $2145.0 b$ & 170.49 & & \\
\hline & 2013 & 1379.0 & 842.5 & 1277.5 & 1315.0 & 1065.0 & 991.2 & 163.87 & & \\
\hline & Mean & $2252.0 \mathrm{a}$ & 1736.3 & 1828.8 & 1966.3 & $1508.8 b$ & $1568.1 b$ & 115.88 & 0.001 & 0.098 \\
\hline \multirow[t]{2}{*}{ May } & 2012 & 2750.0 & 2382.5 & 2215.0 & 2122.5 & 1917.0 & 2027.5 & 161.84 & & \\
\hline & 2013 & 2567.1 & 2642.8 & 2852.5 & 2930.0 & 2310.0 & 2552.5 & 173.02 & & \\
\hline
\end{tabular}




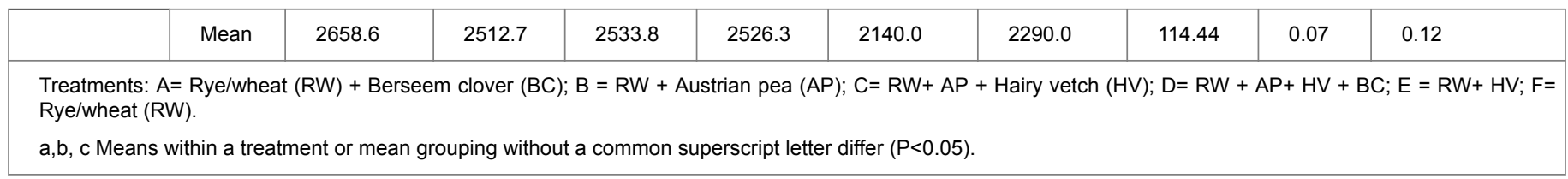

\section{Carcass measurement}

Final BW was obtained after 83 days (about 1 year old), and goats were transported approximately $200 \mathrm{~km}$ to the Fort Valley State University Meat Science Laboratory, GA and kept overnight. On the next morning, before slaughter, goats were weighted after $24 \mathrm{~h}$ without feed (fasting BW) and were harvested according to the USDA approved guidelines [8]. Postmortem necropsy examination and dissection of the kidney and liver were according to the method of [9]. Hot carcass weight ( $\mathrm{HCW}$ ) was determined on the day of slaughter, and carcasses were chilled at $4^{\circ} \mathrm{C}$ for $24 \mathrm{~h}$. Then, cold carcass weight (CCW) and carcass shrink weight were measured. Fat depth over the midpoint of the longissimus muscle (LM) at the 12th rib, measured at the lower point of the12th rib, kidneypelvic fat (KPF), leg circumference, sirloin, loin, shoulder, ribs, hind shank, bones, and trim weight were determined by a certified USDA grader $24 \mathrm{~h}$ postmortem.

The samples from intramuscular fat, subcutaneous adipose muscle and mescentric kidney tissue (MKT) postmortem were taken for fatty acid analysis. Samples of subcutaneous adipose tissue from the region of the ninth rib were vacuum-packed and stored at $-20^{\circ} \mathrm{C}$ until analysis. Fatty acids profile was determined by gas chromatography (GC; HP 5890 Series II, autosampler 7673, HP 3365 ChemStation; Hewlett-Packard Co., Avondale, PA, USA) with a flame ionization detector in accordance with procedures established by [10].

\section{Laboratory measurements}

Analytical DM concentration of forage samples was determined by oven drying at $105^{\circ} \mathrm{C}$ for $3 \mathrm{~h}$ [11], and organic matter (OM) was determined by ashing at $550^{\circ} \mathrm{C}$ for $5 \mathrm{~h} \mathrm{[11].}$ Ash, ether extract (crude fat), total digestible nutrient (TDN), and minerals were analyzed according to the methods described by [11]. Concentration of $\mathrm{N}$ was determined using an organic elemental analyzer [11]. Concentrations of NDF and ADF were sequentially determined using an ANKOM200/220 Fiber Analyzer (ANKOM Technology, Macedon, NY, USA) according to the methodology supplied by the company, which is based on the methods described by [12]. Sodium sulfite was used in the procedure for NDF determination and pre-treated with heat stable amylase (Type XI-A from Bacillus subtilis; Sigma-Aldrich Corporation, St. Louis, MO, USA). Ether extract was measured [11] using a fat analyzer (XT20, ANKOM Technology).

\section{Statistical analyses}

Data were analyzed using the MIXED procedure of SAS (SAS, Inst., Inc., Cary, NC). Data are presented as mean values and the associated SEM. The model included forage bio-mass production, animal performance, carcass quality, fatty acids composition, soil fertility, and associated interactions. Pasture was the experimental units. Year and sampling date of forages were considered random effects. However, carcass traits and soil nutrient compositions were measured at the end of trial in 2012. Chemical compositions of forage samples were measured combined samples from year 2012 and 2013.

\section{Results}

\section{Forage biomass changes}

An average multi-culture forage $\mathrm{DM}(\mathrm{kg} / \mathrm{DM} / \mathrm{ha})$ production during 2 years tended to be $(p=0.07)$ greater for $R W+B C$ than other forage combinations. Biomass production in February and April were higher $(\mathrm{p}<0.01)$ for $\mathrm{RW}+\mathrm{BC}$ than $\mathrm{RW}+\mathrm{HV}$ and RW forage combinations (Table 1). However, forage DM production in March and May were not different among forage combinations. There was no forage treatment $x$ year interactions in the present study.

\section{Forage chemical composition}

Forage chemical composition of multi-cultural forage system is presented in Table 2. There was a forage sampling time $x$ forage treatment interaction $(p<0.01)$ for CP and NDF (data not shown in text). Forage CP content was higher in March than February and April, but DM and NDF content continuously increased with time. The data indicated that plant maturity and forage growth are important factors affecting forage quality and forage biomass production. Interestingly, RW + HV, $\mathrm{RW}+\mathrm{HV}+\mathrm{AP}$, and $\mathrm{RW}+\mathrm{AP}+\mathrm{HV}+\mathrm{BC}$ combinations continued to have greater $(p<0.02) C P$ content compared to others. However, NDF and ADF contents were higher for RW + BC than for other combinations.

Table 2: Forage chemical composition (\% DM) in multi forages system in winter 2012-2013.

\begin{tabular}{|l|l|l|l|l|l|l|l|}
\hline \multirow{2}{*}{ Item } & \multicolumn{2}{|l|}{ Treatment } & \multicolumn{2}{|c|}{ P-value } \\
\cline { 2 - 8 } & A & B & C & D & E & SEM \\
\hline $\mathrm{CP}$ & $15.3 \mathrm{c}$ & $17.8 \mathrm{~b}$ & $18.4 \mathrm{a}$ & $19.9 \mathrm{a}$ & $18.9 \mathrm{~b}$ & $15.5 \mathrm{c}$ & 0.74 \\
\hline
\end{tabular}




\begin{tabular}{|c|c|c|c|c|c|c|c|c|}
\hline ADF & $33.9 a$ & $32.8 a$ & 32.0ab & $32.8 a$ & $31.8 b$ & $32.1 \mathrm{ab}$ & 0.59 & 0.02 \\
\hline NDF & $56.2 a$ & $53.5 a b$ & $52.1 \mathrm{~b}$ & $52.1 \mathrm{~b}$ & $50.1 \mathrm{~b}$ & $52.9 a b$ & 1.14 & 0.01 \\
\hline Crude fat & $3.3 b$ & $3.4 a b$ & $3.5 a b$ & $3.5 a b$ & $3.6 a$ & $3.4 a b$ & 0.09 & 0.01 \\
\hline TDN & $60.8 b$ & $61.5 a b$ & 61.9ab & 61.2ab & $62.1 \mathrm{a}$ & $62.6 a$ & 0.50 & 0.01 \\
\hline Ash & $7.0 \mathrm{~b}$ & 7.5ab & $7.2 a b$ & $7.5 a b$ & $7.7 a$ & $7.1 b$ & 0.18 & 0.03 \\
\hline $\mathrm{Ca}$ & $0.57 b$ & $0.59 b$ & $0.60 a b$ & $0.66 a$ & $0.63 a b$ & $0.51 b$ & 0.02 & 0.01 \\
\hline$P$ & 0.42 & 0.43 & 0.41 & 0.47 & 0.46 & 0.40 & 0.02 & 0.12 \\
\hline $\mathrm{Mg}$ & $0.33 a$ & $0.34 a$ & $0.33 a$ & $0.33 a$ & $0.30 \mathrm{ab}$ & $0.26 \mathrm{~b}$ & 0.01 & 0.001 \\
\hline K & 1.94 & 2.02 & 1.83 & 2.04 & 2.13 & 2.10 & 0.07 & 0.45 \\
\hline$S$ & $0.21 \mathrm{~b}$ & $0.22 a b$ & $0.23 a$ & $0.23 a$ & $0.23 a$ & $0.21 b$ & 0.05 & 0.01 \\
\hline $\mathrm{Na}$ & 0.02 & 0.02 & 0.02 & 0.01 & 0.02 & 0.02 & 0.002 & 0.84 \\
\hline $\mathrm{Cl}$ & 0.72 & 0.78 & 0.75 & 0.75 & 0.74 & 0.74 & 0.04 & 0.30 \\
\hline $\mathrm{Fe}, \mathrm{ppm}$ & 86.8 & 99.6 & 88.1 & 100.0 & 96.1 & 87.0 & 5.21 & 0.08 \\
\hline $\mathrm{Cu}, \mathrm{ppm}$ & 3.5 & 3.3 & 3.5 & 3.7 & 3.6 & 3.5 & 0.20 & 0.39 \\
\hline $\mathrm{Mn}, \mathrm{ppm}$ & $139.1 \mathrm{bc}$ & $130.1 \mathrm{bc}$ & $128.3 \mathrm{c}$ & $157.4 b$ & $156.8 \mathrm{~b}$ & $174.8 a$ & 10.71 & 0.02 \\
\hline Zn, ppm & 21.3 & 20.0 & 20.2 & 23.3 & 23.1 & 20.8 & 1.23 & 0.43 \\
\hline Lysine & $0.21 \mathrm{~b}$ & $0.24 a b$ & $0.25 a b$ & $0.27 a$ & $0.26 a$ & $0.21 \mathrm{~b}$ & 0.02 & 0.001 \\
\hline \multicolumn{9}{|c|}{$\mathrm{CP}=$ crude protein; $\mathrm{ADF}=$ acid detergent fiber; NDF = Neutral detergent fiber; } \\
\hline \multicolumn{9}{|c|}{$\begin{array}{l}\text { Treatments: } A=\text { Rye/wheat }(R W)+\text { Berseem clover }(B C) ; B=R W+\text { Austrian pea }(A P) ; C=R W+A P+\text { Hairy vetch }(H V) ; D=R W+A P+H V+B C ; E=R W+H V ; F= \\
\text { Rye/wheat }(R W) \text {. }\end{array}$} \\
\hline \multicolumn{9}{|c|}{$a, b, c$ Means within a treatment or mean grouping without a common superscript letter differ $(p<0.05)$. } \\
\hline
\end{tabular}

\section{Animal performance and carcass production}

In the present study, animal BW changes and ADG in multiforage system in 2011 and 2012 are presented in Table 3, while carcass characteristics are presented in Table 4. There were no differences in initial BW, final BW and ADG among treatments. There were no treatment $x$ year interactions for animal BW changes and ADG. However, hot carcass weight, cold carcass weight, and dressing percentage were greater $(p<0.05)$ for RW $+\mathrm{BC}$ and $\mathrm{RW}+\mathrm{HV}+\mathrm{AP}$ than for RW grasses (Table 4). Sirloin weight was greater $(p<0.04)$ for $R W+B C$ than for RW pasture, while leg weight was greater $(p<0.03)$ for $R W+H V+A P$ than for RW control.

Table 3: The initial body weight (BW), final body weight, and average daily gain (ADG) in multi-forage system grazing in goats.

\begin{tabular}{|c|c|c|c|c|c|c|c|c|c|c|}
\hline \multirow[t]{2}{*}{ Item } & \multirow[t]{2}{*}{ Year } & \multicolumn{9}{|c|}{ Treatments (Tret) } \\
\hline & & A & B & C & D & $\mathbf{E}$ & $\mathbf{F}$ & SEM & P-value & $\begin{array}{lcr}\text { Tret } \quad x & \text { year } \\
\text { interactions } & \end{array}$ \\
\hline $\mathrm{N}$ & & 8 & 8 & 8 & 8 & 8 & 8 & & & \\
\hline \multirow[t]{3}{*}{ Initial BW } & 2012 & 18.9 & 19.8 & 22.9 & 20.3 & 21.7 & 18.9 & 1.25 & 0.87 & \\
\hline & 2013 & 32.4 & 33.3 & 30.5 & 36.1 & 33.3 & 33.3 & 1.87 & 0.98 & \\
\hline & Mean & 25.7 & 25.6 & 26.7 & 28.2 & 27.5 & 26.1 & 1.25 & 0.78 & 0.31 \\
\hline \multirow[t]{3}{*}{ Final BW } & 2012 & 27.4 & 28.8 & 31.2 & 26.0 & 29.0 & 26.1 & 0.81 & 0.59 & \\
\hline & 2013 & 43.7 & 44.4 & 41.9 & 46.4 & 41.1 & 41.1 & 0.73 & 0.75 & \\
\hline & Mean & 35.5 & 36.6 & 36.6 & 36.1 & 35.0 & 33.6 & 1.32 & 0.59 & 0.22 \\
\hline \multirow[t]{2}{*}{ ADG } & 2012 & 169.4 & 180.2 & 166.0 & 114.8 & 146.6 & 143.2 & 20.38 & 0.83 & \\
\hline & 2013 & 170.4 & 167.5 & 174.3 & 155.2 & 118.5 & 118.5 & 18.88 & 0.71 & \\
\hline
\end{tabular}




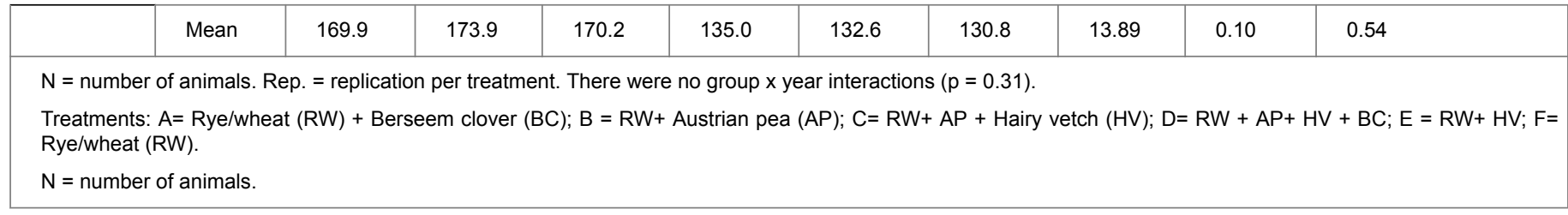

Table 4: Carcass traits (kg/head) of goats grazing in multi forages system in winter 2012.

\begin{tabular}{|c|c|c|c|c|c|c|c|c|}
\hline \multirow[t]{2}{*}{ Item } & \multicolumn{7}{|c|}{ Treatment } & \multirow[t]{2}{*}{ P-value } \\
\hline & A & B & C & D & $E$ & $\mathbf{F}$ & SEM & \\
\hline N1 & 8 & 8 & 8 & 8 & 8 & 8 & & \\
\hline Hot carcass & $12.8 a$ & $12.7 \mathrm{a}$ & $13.2 \mathrm{a}$ & 11.1ab & $12.1 \mathrm{ab}$ & $10.8 b$ & 0.81 & 0.05 \\
\hline Cold carcass & $12.2 \mathrm{a}$ & $11.6 a b$ & $12.6 a$ & $10.5 b$ & $11.8 a b$ & $10.2 b$ & 0.73 & 0.02 \\
\hline Dressing \%2 & $44.0 a$ & $40.0 \mathrm{~b}$ & $48.4 a$ & $39.8 b$ & $41.8 b$ & $41.7 b$ & 0.954 & 0.05 \\
\hline Shoulder & 2.6 & 2.3 & $2.6 a$ & 2.2 & $2.1 \mathrm{~b}$ & $2.0 \mathrm{~b}$ & 0.27 & 0.05 \\
\hline Ribs & 0.98 & 0.97 & 1.03 & 0.81 & 0.84 & 0.81 & 0.08 & 0.08 \\
\hline Loin & 0.85 & 0.85 & 0.91 & 0.76 & 0.77 & 0.74 & 0.07 & 0.31 \\
\hline Sirloin & $0.93 a$ & 0.78 & 0.87 & 0.74 & 0.74 & $0.66 \mathrm{~b}$ & 0.08 & 0.04 \\
\hline Leg & 2.04 & 1.91 & $2.08 a$ & 1.69 & 1.93 & $1.67 \mathrm{~b}$ & 0.12 & 0.03 \\
\hline Hind shank & 0.47 & 0.46 & 0.53 & 0.46 & 0.61 & 0.46 & 0.07 & 0.07 \\
\hline Trim & 0.34 & 0.31 & 0.35 & $0.27 \mathrm{~b}$ & $0.37 a$ & 0.31 & 0.04 & 0.04 \\
\hline Bones & 0.39 & 0.35 & 0.35 & 0.34 & 0.42 & 0.30 & 0.03 & 0.05 \\
\hline Kidney fat & 0.09 & 0.09 & 0.10 & 0.07 & 0.12 & 0.07 & 0.034 & 0.24 \\
\hline \multicolumn{9}{|c|}{ a,b, c Means within a treatment or mean grouping without a common superscript letter differ $(p<0.05)$. } \\
\hline \multicolumn{9}{|c|}{$\begin{array}{l}\text { Treatments: } A=\text { Rye/wheat }(R W)+\text { Berseem clover }(B C) ; B=R W+\text { Australian pea }(A P) ; C=R W+A P+\text { Hairy vetch }(H V) ; D=R W+A P+H V+B C ; E=R W+H V ; F= \\
\text { Rye/wheat }(R W) \text {. }\end{array}$} \\
\hline \multicolumn{9}{|c|}{2 Dressing percentage $=(\text { hot carcass weight } / L W)^{\star} 100}$. \\
\hline
\end{tabular}

\section{Fatty acids profile in carcass}

Carcass fatty acids profiles in intramuscular, mescentric kidney, and sub-cutaneous fats are presented in Tables 5-7. Goats grazed on grasses-based diet (RW), had higher saturated fatty acids (SFA; $p<0.01$ ) and polyunsaturated fatty acids (PUFA; $\mathrm{p}<0.05$ ) in intramuscular fat content, but both were lower in mescentric kidney fat (MKF) and subcutaneous fat $(p<0.05-0.01)$ compared to legume forage-based diets (Tables
5-7). Goats grazed on grass-based diets, had higher omega-3 and -6 fatty acids in intramuscular fat content $(p<0.05-0.08)$, but both were lower in subcutaneous fat $(p<0.05-0.01)$ compared to legume forage-based diets. Mono unsaturated fatty acids (MFA) were not affected by dietary forage combination. These results indicated that goats receiving grass-based diets produced carcasses with more PUFA and higher omega- 3 and -6 fatty acids in intramuscular fat from Kiko-crossbred male goats.

Table 5: Effects of legume and grass forage on fatty acids composition (mg/g of tissue) of intramuscular fat from Kiko crossbred male goat kids

\begin{tabular}{|l|l|l|l|l|}
\hline \multirow{2}{*}{ Item } & \multicolumn{2}{|l|}{ Diets } & SEM & p-value \\
\cline { 2 - 5 } & Wheat \& Rye & Legume forage & & \\
\hline Number of animals & 8 & 40 & & \\
\hline C10 & 226.6 & 33.5 & 163.9 & 0.53 \\
\hline C11 & 140.2 & 155.5 & 65.67 & 0.87 \\
\hline
\end{tabular}




\begin{tabular}{|c|c|c|c|c|}
\hline C12 & 99 & 122.07 & 38.1 & 0.78 \\
\hline C13 & 187.4 & 284.7 & 114.76 & 0.32 \\
\hline C14 & 185.3 & 198.6 & 167.86 & 0.48 \\
\hline C14:1 & 78 & 84.9 & 49.95 & 0.94 \\
\hline C15 & 68.6 & 141.4 & 58.81 & 0.73 \\
\hline C15:1 & 234 & 76.8 & 68.86 & 0.24 \\
\hline C16:0 & 141.4 & 65.3 & 49.9 & 0.33 \\
\hline C16:1 & 36.3 & 20.4 & 18.18 & 0.57 \\
\hline C17:0 & 193.6 & 173.6 & 57.3 & 0.38 \\
\hline C17:1 & 79.8 & 109.9 & 44.6 & 0.66 \\
\hline C18:0 & 116.5 & 82.4 & 40.45 & 0.54 \\
\hline C18:1n9c & 244.8 & 175.4 & 0.39 & 0.39 \\
\hline C18:1n9t & 169.3 & 175.4 & 110.8 & 0.92 \\
\hline C18:2n6c & 111.5 & 101.6 & 30.9 & 0.83 \\
\hline C18:2n6t & 14.9 & 64.6 & 23.18 & 0.15 \\
\hline C18:3n3c & 24.5 & 34.5 & 14.7 & 0.66 \\
\hline C18:3n6 & 193.4 & 56.8 & 79.68 & 0.28 \\
\hline C19 & 65.4 & 74 & 5.6 & 0.33 \\
\hline C2O & 84.4 & 28.4 & 18.8 & 0.07 \\
\hline C20:1 & 70.6 & 86.5 & 33.21 & 0.75 \\
\hline C20:3n3 & 27.9 & 19.5 & 11.24 & 0.61 \\
\hline C20:3n6 & 79.4 & 55.5 & 36.41 & 0.67 \\
\hline C20:4n6 & 32.7 & 27.4 & 20.071 & 0.86 \\
\hline C20:5n3 & 432.4 & 161.4 & 178.21 & 0.33 \\
\hline $\mathrm{C} 21$ & 44.5 & 55.8 & 17.63 & 0.07 \\
\hline $\mathrm{C} 22$ & 47.3 & 175.7 & 44.55 & 0.08 \\
\hline $\mathrm{C} 22: 2$ & 257.4 & 240.2 & 98.7 & 0.91 \\
\hline C22:6n3 & 779.6 & 692.8 & 282.1 & 0.84 \\
\hline $\mathrm{C} 23$ & 73.5 & 23.5 & 24.22 & 0.19 \\
\hline $\mathrm{C} 24$ & 377.5 & 315.8 & 191.71 & 0.83 \\
\hline $\mathrm{C} 24: 1$ & 878.6 & 418.9 & 322.4 & 0.36 \\
\hline SFA & 527.6 & 374.7 & 29.01 & 0.01 \\
\hline MFA & 564.5 & 547.2 & 36.9 & 0.71 \\
\hline PUFA & 916.7 & 521.3 & 33.11 & 0.05 \\
\hline PUFA/SFA & 1.74 & 1.39 & 0.35 & 0.18 \\
\hline Total omega-3 & 1204.4 & 908.2 & 180.77 & 0.08 \\
\hline Total omega- 6 & 352.4 & 250.4 & 133.6 & 0.05 \\
\hline \multicolumn{5}{|c|}{$S F A=$ saturated fatty acid $=(C 14: 0+C 16: 0+C 18: 0+C 20: 0)$} \\
\hline \multicolumn{5}{|c|}{ MFA=Monounsaturated fatty acid $=(C 17: 1+C 18: 1+C 20: 1)$} \\
\hline
\end{tabular}


PUFA- polyunsaturated fatty acid $=(C 18: 2+C 18: 3+C 20: 2+C 20: 3+C 20: 4+C 22: 4)$

Table 6: Effects of legume and grass forage on fatty acids composition (mg/g of tissue) of mescentric kidney fat (MKF) from Kiko crossbred male goat kids.

\begin{tabular}{|c|c|c|c|c|}
\hline \multirow[t]{2}{*}{ Item } & \multicolumn{2}{|l|}{ Diets } & \multirow[t]{2}{*}{ SEM } & \multirow[t]{2}{*}{ p-value } \\
\hline & Wheat \& Rye & $\begin{array}{l}\text { Legume } \\
\text { forage }\end{array}$ & & \\
\hline Number of animals & 8 & 40 & & \\
\hline C10 & 150.7 & 73.6 & 51.11 & 0.3 \\
\hline C11 & 465.8 & 193.1 & 201.7 & 0.33 \\
\hline C12 & 333.6 & 258.3 & 191.9 & 0.78 \\
\hline C13 & 398 & 316.9 & 180.3 & 0.75 \\
\hline C14 & 500.1 & 322.7 & 188.64 & 0.6 \\
\hline C14:1 & 177.2 & 254.9 & 120.74 & 0.61 \\
\hline C15 & 206.7 & 164.1 & 79.9 & 0.76 \\
\hline C15:1 & 60.3 & 81.1 & 25.34 & 0.03 \\
\hline C16 & 880.1 & 894.5 & 267.3 & 0.96 \\
\hline C16:1 & 189.5 & 101.1 & 18.18 & 0.35 \\
\hline C17 & 77.3 & 388.8 & 98.3 & 0.04 \\
\hline C17:1 & 114.6 & 135.4 & 44.6 & 0.63 \\
\hline C18 & 1387.4 & 2129.7 & 600.2 & 0.32 \\
\hline C18:1n9c & 1252.3 & 1524.3 & 424.8 & 0.61 \\
\hline C18:1n9t & 231.5 & 221.6 & 71.9 & 0.92 \\
\hline $18: 2 n 6 c$ & 139.3 & 264.1 & 51.4 & 0.01 \\
\hline C18:2n6t & 27.4 & 82.9 & 41.5 & 0.03 \\
\hline C18:3n3 & 99.2 & 34.4 & 30.9 & 0.2 \\
\hline C18:3n6 & 170.3 & 123.8 & 67.15 & 0.02 \\
\hline C19 & 74 & 78.2 & 3.5 & 0.66 \\
\hline $\mathrm{C} 20$ & 64.9 & 183.1 & 96.15 & 0.001 \\
\hline $\mathrm{C} 20: 1$ & 171.1 & 39.2 & 85.1 & 0.69 \\
\hline C20:3n3 & 23.1 & 11.9 & 8.01 & 0.88 \\
\hline C20:3n6 & 49.7 & 32.8 & 29.2 & 0.037 \\
\hline C20:4n6 & 60.3 & 60.1 & 24.4 & 0.68 \\
\hline C20:5n3 & 80.6 & 161.4 & 178.21 & 0.33 \\
\hline $\mathrm{C} 21$ & 144.5 & 51.5 & 42.63 & 0.01 \\
\hline $\mathrm{C} 22$ & 47.3 & 175.7 & 44.55 & 0.08 \\
\hline $\mathrm{C} 22: 2$ & 148.9 & 142.4 & 55.7 & 0.39 \\
\hline C22:6n3 & 530.5 & 425.7 & 116.76 & 0.71 \\
\hline $\mathrm{C} 23$ & 31.9 & 76 & 47.4 & 0.39 \\
\hline $\mathrm{C} 24$ & 32.7 & 238.2 & 92.51 & 0.001 \\
\hline
\end{tabular}




\begin{tabular}{|c|c|c|c|c|}
\hline C24:1 & 449.1 & 303.1 & 81.86 & 0.95 \\
\hline SFA & 2832.5 & 3530 & 362.11 & 0.01 \\
\hline MFA & 1769.5 & 1920.5 & 359 & 0.29 \\
\hline PUFA & 649.9 & 771.4 & 25.03 & 0.05 \\
\hline Total omega-3 & 733.4 & 633.4 & 110.23 & 0.11 \\
\hline Total omega- 6 & 419.6 & 480.8 & 115.4 & 0.24 \\
\hline
\end{tabular}

Table 7: Effects of legume and grass forage on fatty acids composition (mg/g of tissue) of sub-cutaneous fat from Kiko crossbred male goat kids

\begin{tabular}{|c|c|c|c|c|}
\hline \multirow[t]{2}{*}{ Item } & \multicolumn{2}{|l|}{ Diets } & \multirow[t]{2}{*}{ SEM } & \multirow[t]{2}{*}{ p-value } \\
\hline & Wheat \& Rye & Legume forage & & \\
\hline Number of animals & 8 & 20 & & \\
\hline C10 & 91.1 & 30.9 & 25.28 & 0.20 \\
\hline C11 & 46.9 & 66.5 & 21.1 & 0.67 \\
\hline C12 & 122.4 & 62.9 & 48.95 & 0.28 \\
\hline C13 & 59.4 & 275.8 & 124.8 & 0.36 \\
\hline $\mathrm{C} 14$ & 217.9 & 421.4 & 121.1 & 0.22 \\
\hline C14:1 & 156.0 & 374.1 & 227.52 & 0.47 \\
\hline C15 & 31.4 & 283.9 & 119.42 & 0.28 \\
\hline C15:1 & 818.2 & 503.1 & 489.51 & 0.89 \\
\hline C16 & 509.9 & 902.7 & 215.04 & 0.21 \\
\hline C16:1 & 334.7 & 344.4 & 129.31 & 0.72 \\
\hline C17 & 45.4 & 157.4 & 39.94 & 0.18 \\
\hline C17:1 & 591.5 & 623.8 & 534.55 & 0.76 \\
\hline C18 & 568.5 & 1080.4 & 383.22 & 0.16 \\
\hline C18:1n9c & 1060.1 & 2622.6 & 342.7 & 0.05 \\
\hline C18:1n9t & 2689.2 & 288.4 & 281.70 & 0.002 \\
\hline $18: 2 n 6 c$ & 233.4 & 235.9 & 92.8 & 0.93 \\
\hline $\mathrm{C} 18: 2 \mathrm{n} 6 \mathrm{t}$ & 180.2 & 67.7 & 82.8 & 0.12 \\
\hline C18:3n3 & 13.4 & 166.5 & 62.91 & 0.27 \\
\hline C18:3n6 & 75.1 & 123.3 & 95.04 & 0.51 \\
\hline C19 & 74.0 & 78.2 & 3.50 & 0.66 \\
\hline $\mathrm{C} 20$ & 4.9 & 232.2 & 44.61 & 0.05 \\
\hline C20:1 & 44.3 & 131.5 & 85.3 & 0.15 \\
\hline C20:3n3 & 3.6 & 130.9 & 34.71 & 0.05 \\
\hline C20:3n6 & 44.4 & 205.4 & 96.8 & 0.09 \\
\hline $\mathrm{C} 20: 4 \mathrm{n} 6$ & 7.8 & 61.7 & 29.7 & 0.06 \\
\hline C20:5n3 & 65.1 & 75.1 & 39.56 & 0.81 \\
\hline
\end{tabular}




\begin{tabular}{|l|l|l|l|l|}
\hline $\mathrm{C} 21$ & 75.4 & 84.5 & 23.5 & 0.84 \\
\hline $\mathrm{C} 22$ & 165.3 & 368.7 & 104.6 & 0.05 \\
\hline $\mathrm{C} 22: 2$ & 73.1 & 143.4 & 31.54 & 0.05 \\
\hline $\mathrm{C} 22: 6 \mathrm{n3}$ & 263.3 & 1292.4 & 116.76 & 0.05 \\
\hline $\mathrm{C} 23$ & 39.1 & 44.4 & 18.8 & 0.95 \\
\hline $\mathrm{C} 24$ & 139.9 & 58.1 & 43.32 & 0.24 \\
\hline $\mathrm{C} 24: 1$ & 506.7 & 353.1 & 216.99 & 0.75 \\
\hline SFA & 1301.2 & 2636.7 & 190.99 & 0.01 \\
\hline MFA & 4385.1 & 3666.3 & 311.06 & 0.29 \\
\hline PUFA & 623.1 & 1066.5 & 66.78 \\
\hline Total omega-3 & 345.4 & 1664.9 & 63.48 \\
\hline Total omega-6 & 540.9 & 694.0 & 79.42 \\
\hline $\begin{array}{l}\text { SFA saturated fatty acid }=(\mathrm{C} 14: 0+\mathrm{C} 16: 0+\mathrm{C} 18: 0+\mathrm{C} 20: 0) . \\
\text { MFA=Monounsaturated fatty acid }=(\mathrm{C} 17: 1+\mathrm{C} 18: 1+\mathrm{C} 20: 1) .\end{array}$ & & 0.05 \\
\hline PUFA- polyunsaturated fatty acid $=(\mathrm{C} 18: 2+\mathrm{C} 18: 3+\mathrm{C} 20: 2+\mathrm{C} 20: 3+\mathrm{C} 20: 4+\mathrm{C} 22: 4)$. & & 0.01 \\
\hline
\end{tabular}

\section{Discussion}

It has been reported that berseem clover is one of the fast growing winter legume forage and can produce up to 3.5 tons per ha of biomass under irrigation [13]. Berseem clover also stablishes well with an oat nurse crop, making it an excellent cover for small grain>corn>soybean rotations [14] Ghaffarzadeh [14] reported that oat under seeded with berseem clover produced up to $70 \%$ more biomass (without grain) than sole-crop oat. Current study showed that biomass production in February and April were higher $(p<0.01)$ for RW $+\mathrm{BC}$ than RW + HV and RW forage combinations. Therefore, grasses with legume forage combination had potential benefits to produce maximum biomass for forage-based goat production system.

The data indicated that plant maturity and forage growth are important factors affecting forage biomass production and quality. Forage $\mathrm{CP}$ content was higher in March than February and April, but DM and NDF content continuously increased with time. Interestingly, $\mathrm{RW}+\mathrm{HV}, \mathrm{RW}+\mathrm{HV}+\mathrm{AP}$, and $\mathrm{RW}+\mathrm{AP}+$ $\mathrm{HV}+\mathrm{BC}$ combinations continued to have greater $\mathrm{CP}$ content compared to others. However, NDF and ADF contents were higher for $\mathrm{RW}+\mathrm{BC}$ than for other combinations. In agreement with our results, [15] and [16] reported greater CP content in annual legume based diet than grasses. The crude fat and TDN content were greater $(\mathrm{P}<0.01)$ for RW $+\mathrm{HV}$ and RW than for other combinations. Forage mineral contents were varied among forage treatments.

In the present study, there were no differences in initial BW, final $B W$ and $A D G$ among treatments with no treatment $x$ year interactions for animal BW changes and ADG. However, hot carcass weight, cold carcass weight, and dressing percentage were greater for $\mathrm{RW}+\mathrm{BC}$ and $\mathrm{RW}+\mathrm{HV}+\mathrm{AP}$ than for $\mathrm{RW}$ grasses. Sirloin weight was greater for $\mathrm{RW}+\mathrm{BC}$ than for RW pasture, while leg weight was greater $(p<0.03)$ for $\mathrm{RW}+\mathrm{HV}+$
AP than for RW control. Values reported for improved animal performance and carcass yield with legume foragesin the present study are consistent with other researchers $[17,18]$. Wildeus [18] reported that Spanish goat kids fed alfalfa-hay had higher cold carcass weight $(13 \%)$ and dressing percent (4\%), but similar ribeye area, back fat and kidney/pelvic fat compared to the grass-hay-fed kids. It has been reported that increasing CP concentration of diets resulted in the higher carcass yield and dressing percentage in wether goats $[18,19]$. Similarly in the present study, legume forages-based diets having the higher $\mathrm{CP}$ concentration caused to the higher dressing percentage. Wuliji [20] also reported an increased ribeye area in alfalfa-hay-fed compared to grass-hay-fed Spanish meat goats, which we did not observe.

Fatty acids are the major component of lipids and affect meat quality. The fatty acid composition of fats determines its degree of saturation, and therefore, significantly affects its quality. Goats grazed on grasses-based diet (RW) in the present study had higher SFA and PUFA in intramuscular fat content, but both were lower in MKF and subcutaneous fat compared to legume forage-based diets. Finishing beef on forages generally leads to a more favorable fatty acid profile compared to concentrate-finished ruminant meat. Perhaps most important to human health, total fat content is reduced when beef is finished on forages compared to beef finished on concentrates to a similar endpoint, reducing SFA, MUFA, and omega-6 FA on a per serving basis [21].

Numerous studies have established differences in fatty acid deposition between beef finished on forage versus concentrate diets. How specific forages (grasses vs. legume forages) alter the deposition of fatty acids and antioxidants must continue to be studied. Different forages provide different lipids and fatty acids profile in the ruminant diet. In ryegrass, C18:3 represent 55 to $66 \%$ of total fatty acids but only $40 \%$ in alfalfa [22]. Lough and Anderson [23] reported 
that C18:3 was the major component of esterified fatty acids (simple and complex lipids) in mixed pasture grasses including ryegrass (Lolium multiflorum Lam.), timothy (Phleum pratense L.), and meadow fescue (Festuca pratensis Huds.). It has been reported that an annual ryegrass cultivar had the highest C18:3 concentrations $(20.6 \mathrm{mg} / \mathrm{g} \mathrm{DM})$ than timothy $(7.3 \mathrm{mg} / \mathrm{g}$ DM) $[22,23]$. Among legumes, white clover (Trifolium repens L.) cultivar had the highest C18:3 concentration $(16.5 \mathrm{mg} / \mathrm{g}$ DM) whereas alfalfa (Medicago sativa L.) cultivar had the lowest $(6.0 \mathrm{mg} / \mathrm{g} \mathrm{DM}$; [24]. Goats grazed on grass-based diets in the present study had higher omega- 3 and -6 fatty acids in intramuscular fat content, but both were lower in subcutaneous fat compared to legume forage-based diets. Mono unsaturated fatty acids (MFA) were not affected by dietary forage combination. These results indicated that goats receiving grass-based diets produced carcasses with more PUFA and higher omega- 3 and -6 fatty acids in intramuscular fat from Kiko-crossbred male goats [25] reported that CLA cis-9, trans-11 concentration was greater in bermudagrass and pearl millet than alfalfa, chicory, or cowpea forages. Chicory and cowpea treatments had greater concentrations of linolenic acid than other treatments, whereas alfalfa was higher in concentration of linolenic acid than pearl millet.

\section{Conclusion}

In the study, it has been reported that PUFA and n- 6 fatty acids of intra muscular fat were higher in goats receiving grassbased diets as seen in Table 5 . However, in conclusion, it has been started that the goats receiving grass-legume multiple combination had the more desirable fatty acids composition. It could not be concluded from the results of this study.

\section{Conflict of Interest}

No known conflict of interest

\section{Acknowledgement}

The authors thank the George Washington Carver Agricultural Experiment Station, Tuskegee University and USDA Southern Region Sustainable Agriculture Research and Education grant (S-SARE; RD309-105/4690178).

\section{References}

1. Horn GW (1994) Proc. of Wheat land Stocker Conf. Enid, Oklahoma: E-1.

2. Poore MH, Drewnoski ME (2010) Review: Utilization of stockpiled tall fescue in winter grazing systems for beef cattle. The Prof Anim Scient 26: 142-149.

3. Poore $\mathrm{MH}$, Drewnoski ME (2010) Review: Utilization of stockpiled tall fescue in winter grazing systems for beef cattle. The Prof Anim Scient 26: 150-155.

4. Schmidt JR, Miller MC, Andrae JG, Ellis SE, Duckett SK, et al. (2013) Effect of summer forage species grazed during finishing on animal performance, carcass quality, and meat quality. J Anim Sci 91: 4451-4461.
Kalač $P$ (2011) The effects of feeding fresh forage and silage on some nutritional attributes of beef: an overview. J Agrobiol 28: $1-13$.

6. Givens DI, Kliem KE, Gibbs R.A (2006) The role of meat as a source of n-3 polyunsaturated fatty acids in the human diet. Meat Sci 74: 209-218.

7. Duckett SK, Neel JPS, Lewis RM, Fontenot JP, Clapham WM, et al. (2013) Effects of forage specieas or concentrate finishing on animal performance, carcass and meat quality. J Anim Sci 91: 1454-1467.

8. USDA (2001) Institutional meat purchased specifications for fresh goat. Series 11. USDA, MRP, AMF, Livestock and Seed Program, Washington, DC.

9. Nietfeld JC (2010) Field necropsy techniques and proper specimen submission for investigation of merging infectious diseases of food animals. Vet Clin North Am Food Anim Pract 26: $1-13$.

10. Noci F, French P, Monahan FJ, Moloney AP (2007) The fatty acid composition of muscle fat and subcutaneous adipose tissue of grazing heifers supplemented with plant oil-enriched concentrates. J Anim Sci 85: 1062-1073.

11. AOAC (2000) Official Methods of Analysis. (17thedn) Association of Official Analytical Chemists, Gaithersburg, MD, USA.

12. Van Soest PJ, Robertson JB, Lewis BA, (1991) Methods for dietary fiber, neutral detergent fiber, and non-starch polysaccharides in relation to animal nutrition. J Dairy Sci 74: 473-481.

13. Andy C (2007) Managing Cover Crops Profitably. (3rdedn) Sustainable Agriculture Network, Beltsville, MD.

14. Ghaffarzadeh M (1997) Economic and biological benefits of intercropping berseem clover with oats in corn-soybean-oat rotations. J Prod Agric 10: 314-319.

15. Hafley J, Nipper WA, Craig WM, Adkinson RW, Achacoso AS, et al. (1987) In vitro protein degradation of cool season forages planted with minimum tillage and no fertilization. J Dairy Sci 70: 2322-2329.

16. Fraser J, McCartney D, Najda H, Mir Z (2004) Yield potential and forage quality of annual forage legumes in southern Alberta and northeast Saskatchewan. Can J Plant Sci 84: 143-155.

17. Martens JRT, Entz MH (2011) Integrating green manure and grazing systems: A review. Can J Plant Sci 91: 811-824.

18. Wildeus S, Luginbuhl JM, Turner KE, Nutall YL, Collins JR, et al. (2007) Growth and carcass characteristics in goat kinds fed gradd- and alfalfa-hay based diets with limited concentrate supplementation. Sheep and Goats Res J 22: 15-19.

19. Shahialal M, Galbraith H, Topps JH (1992) The effect of changes in dietary protein and energy on growth, body composition and mohair fiber characteristics of British Angora goats. Anim Prod 54: 405-412.

20. Wiliji T, Goetsch AL, Sahlu T, Puchala R, Soto-Navarro S, et al. (2003) Effects of different quality diets consumed continuously or after a lower quality diet on characteristics of growth of young Spanish goats. Small Rumin Res 50: 83-96.

21. Duckett SK, Neel JPS, Fontenot JP, Clapham WM (2009) Effects of winter stocker growth rate and finishing system on: III. Tissue proximate, fatty acid, vitamin, and cholesterol content. J Anim Sci 87: 2961-2970. 
22. Bauchart D, Doreau M, Legay-Carmier F (1985) Utilisation digestive des lipides et conséquences de leur introduction sur la digestion du ruminant. Bull Tech C R Z V 61: 65-77.

23. Lough AK, Anderson LJ (1973) Effect of ensilage on the lipids of pasture grasses. Proc Nutr Soc 32: 61A.

24. Boufaïed H, Chouinard PY, Tremblay GF, Petit HV, Michaud R, et al. (2003) Fatty acids in forages. I. Factors affecting concentrations. Can J Anim Sci. 83: 501-51.
25. Schmidt J (2009) Forage species alters animal performance, carcass quality, and fatty acid composition of forage-finished beef produced in summer months. Thesis, Clemson University, SC. 